\title{
Glutathione Pegylated Liposomal Doxorubicin Hydrochloride Formulation 2B3-101
}

National Cancer Institute

\section{Source}

National Cancer Institute. Glutathione Pegylated Liposomal Doxorubicin Hydrochloride

Formulation 2B3-101. NCI Thesaurus. Code C106121.

A glutathione (GSH) pegylated, liposome-encapsulated preparation of the hydrochloride salt form of the anthracycline antineoplastic antibiotic doxorubicin, with potential anetineoplastic activity. Upon administration, the glutathione pegylated liposomal formulation 2B3-101 specifically delivers doxorubicin into the brain. Doxorubicin intercalates between DNA base pairs and interferes with topoisomerase II activity, which inhibits both DNA replication and RNA synthesis, resulting in cancer cell death and tumor regression. Doxorubicin also generates reactive oxygen species, which causes cell membrane lipid peroxidation leading to cytotoxicity. The pegylated liposomal delivery of doxorubicin improves drug penetration into tumors and prolongs circulation time, thereby increasing doxorubicin's efficacy and decreasing its toxicity. Conjugation of GSH to the PEG molecules directs the liposomes to the GSH transporters on the blood brain barrier (BBB) and improves the delivery of doxorubicin into the brain. 